\title{
Self-Driving Cars: a qualitative study into the opportunities, challenges and perceived acceptability for people with epilepsy
} Mustafa Sultan ${ }^{1}$, Rhys H Thomas ${ }^{2,3}$

1. The Medical School, Newcastle University, Framlington Place, Newcastle-upon-Tyne NE2 $4 \mathrm{HH}$, United Kingdom

2. Department of Neuroscience, Royal Victoria Infirmary, Queen Victoria Road, NewcastleUpon-Tyne, NE1 4LP, United Kingdom

3. Translational and Clinical Research Institute, Henry Wellcome Building, Framlington Place, Newcastle-Upon-Tyne, NE2 4HH, United Kingdom

\section{Address for Correspondence:}

Translational and Clinical Research Institute, Henry Wellcome Building, Framlington Place, Newcastle-Upon-Tyne, NE2 4HH, United Kingdom

rhys.thomas@ncl.ac.uk

\section{Acknowledgments}

The authors would like to thank Epilepsy Action and the CANDO Project for their help with recruiting participants.

\section{Disclosures}

R Thomas has received honoraria from Eisai, GW Pharma, Sanofi, UCB Pharma, Zogenix and meeting support from Bial, LivaNova and Novartis.

Word Count: 996 


\section{Self-Driving Cars: a qualitative study into the opportunities, challenges and perceived acceptability for people with epilepsy}

Driving restrictions faced by people with epilepsy (PWE) represent a crucial and modifiable factor that predicts their social participation and employability. In the case of refractory epilepsy, a person may lose the ability to drive forever - a handicap associated with significant detrimental effects, including reduced employability and a poorer household income (1).

Encouragingly, progress in self-driving car technology provides renewed hope for PWE restricted from driving. A self-driving car is any car in which steering or acceleration/deceleration are controlled by the car. Differing degrees of automation exist, but relevant to PWE are those which require no human input to drive (fully autonomous vehicles). These are vehicles with no steering wheel-and in which, the driver, for all intents and purposes is a passenger.

The UK government aim to have self-driving cars on the road by 2021 (2). Promisingly, Department of Transport guidelines stipulate: "It would seem reasonable to allow ownership or use of a fully automated vehicle without the need to hold a driving license" (3). This could mean that all PWE will be able to 'drive' in the imminent future and although this innovation is exciting, it is important to consider the views of PWE.

\section{Methods}

Eight people with epilepsy (or carers of) were recruited including four men and four women between the ages of 31 and 68. Three detailed semi-structured focus groups were conducted. Recruitment and group interviews were continued until no new themes were identified.

To ensure that all parties were discussing the same form of technology, participants were told that the self-driving cars being discussed would be fully autonomous vehicles (not requiring a steering wheel) and to assume that PWE would be able to operate them without a driving license. With the aid of contemporaneous notes, transcripts were analysed 
Sultan, Thomas Self-driving Cars

independently by the authors using a thematic analysis approach. Newcastle University Ethics Committee grated approval for the project (17242/2018).

\section{Results}

\section{Independence and Guilt}

Many participants were devastated by driving restrictions: “it's like losing a limb - your independence has been taken away" and some felt guilt over asking their support network for transport. Therefore, most participants were cautiously optimistic about the independence that self-driving cars could offer.

\section{Stigma and Concerns Over the Technology}

Many were sceptical of the self-driving cars' ability to detect road hazards as competently as a human driver: "you can look two or three cars ahead and you can see the brake lights going on, on a bus or van. Whereas, if you're in a self-driving car, is the car going to see those brake lights? Is it going to see somebody crossing the road?". In reality, self-driving cars may have a lower accident rate than human operated vehicles (4).

There were also disease-specific concerns surround the self-driving vehicles. Participants questioned what would happen if the 'driver' had a seizure inside the car - a parent was concerned about his daughter's wellbeing whilst she was in the car unsupervised. Participants could not agree on whether they would like the car to continue on its journey or come to a safe stop in the event of the seizure.

Participants worried that the general public would not accept PWE operating self-driving cars due to stigma. They were also concerned that a government scheme to provide PWE self-driving vehicles may provide a specific model of vehicle which would become identifiable as the epilepsy car and be a focus for stigma: "If you've got an invisible illness people don't always know something's wrong, but if you're driving that car people are going to know something's wrong. And a lot of people won't want to put themselves out there in that position". 
Sultan, Thomas Self-driving Cars

\section{Improvements in Disease Management}

Participants identified driving eligibility as a barrier to identifying the optimal drug regimen for their epilepsy. They expressed that both doctors and patients are reluctant to adjust the doses of anti-epileptic drugs (AEDs) once they have reached a 12-month seizure-free period for fear of causing a breakthrough seizure and subsequent driving ineligibility. It was postulated that self-driving cars would give clinicians freedom to down-titrate their AEDs and thus result in fewer drug side effects. 


\section{Discussion}

We recognise some limitations to this study. Epilepsy is a common condition and as a result, it can be challenging to identify participants who fully represent the community. Volunteers are more likely to be professional, have family support networks and be more comfortable communicating in a group. In addition to this, the sample studied was small $(n=8)$ without inclusion of professional drivers whose views may have differed.

Most PWE recognised the potential benefits of driverless vehicle technology. Concerns primarily centred around the practicalities of the technology, with a small minority being firmly opposed to it. To benefit the full spectrum of PWE, car manufacturers will have to consider adaptations that can give parents and carers peace of mind. These could include inward facing cameras and sensors which can be monitored remotely and safety-proofing the car's cabin in case of a seizure.

The onus will be on Governments to ensure that this technology is not just limited to the wealthy but adopted by those it could benefit most. They must ensure that driving legislation closely follows innovation; meaning that PWE are able to operate fully autonomous vehicles without holding a driving license, as well as ensuring that government mobility allowance can fund such vehicles. The impact of self-driving cars will not be limited to just PWE - diseases such as diabetes, cardiac conditions, sleep apnoea and stroke can all prevent people from driving (5).

The freedom that self-driving cars could bring PWE would be transformative for many of our patients. Our participants identified concerns about the technology as citizens, as well as epilepsy-specific anxieties. Nonetheless there will be a significant majority of people with epilepsy, who are currently legally denied their rights to independence and to drive because of legitimate safety concerns, that could have this limitation overturned with a self-driving car. 
Sultan, Thomas Self-driving Cars

\section{References}

1. Bautista RED, Wludyka P. Driving prevalence and factors associated with driving among patients with epilepsy. Epilepsy Behav. 2006;9(4):625-31.

2. Gov.uk. Government moves forward on advanced trials for self-driving vehicles [Internet]. 2019 [cited 2019 Aug 23]. Available from:

https://www.gov.uk/government/news/government-moves-forward-on-advancedtrials-for-self-driving-vehicles

3. The Pathway to Driverless Cars: A detailed review of regulations for automated vehicle technologies [Internet]. 2015. Available from:

https://assets.publishing.service.gov.uk/government/uploads/system/uploads/attach ment_data/file/401565/pathway-driverless-cars-main.pdf

4. Blanco M, Atwood J, Russell S, Trimble T, McClafferty J, Perez M. Automated Vehicle Crash Rate Comparison Using Naturalistic Data [Internet]. Vtti. 2016. Available from: https://vtechworks.lib.vt.edu/bitstream/handle/10919/64420/Automated Vehicle Crash Rate Comparison Using Naturalistic Data_Final Report_20160107.pdf?sequence=1\&isAllowed=y

5. Medical conditions, disabilities and driving [Internet]. Gov.uk. [cited 2019 Aug 23]. Available from: https://www.gov.uk/driving-medical-conditions 